Journal of

Synchrotron

Radiation

ISSN 0909-0495

Received 18 November 2004

Accepted 4 March 2005

C 2005 International Union of Crystallography Printed in Great Britain - all rights reserved

\section{VUV irradiation effects on proteins in high-flux synchrotron radiation circular dichroism spectroscopy}

\author{
F. Wien, ${ }^{a}$ A. J. Miles, ${ }^{\text {a J. G. Lees, }},{ }^{a}$ S. Vrønning Hoffmannn ${ }^{b}$ and B. A. Wallace ${ }^{\mathrm{a}, \mathrm{c} *}$ \\ a Department of Crystallography, Birkbeck College, University of London, London WC1E 7HX, UK, \\ ${ }^{\mathbf{b}}$ Institute for Storage Ring Facilities, University of Aarhus, DK-8000 Aarhus C, Denmark, and \\ ${ }^{\mathbf{c}}$ Centre for Protein and Membrane Structure and Dynamics, Daresbury Laboratory, Warrington \\ WA4 4A, UK. E-mail: ubcg25a@mail.cryst.bbk.ac.uk
}

\begin{abstract}
Synchrotron radiation circular dichroism (SRCD) spectroscopy is emerging as an important new tool in structural molecular biology. Previously we had shown that in lower-flux SRCD instruments, such as UV1 at ISA and beamline 3.1 at the SRS, vacuum ultraviolet (VUV) radiation damage to proteins was not evident after exposure over a period of hours. No effects were detected in either the protein primary or the secondary structures. However, with the development of high-flux beamlines, such as CD12 at the SRS, this issue has been revisited because of changes observed in the SRCD spectra of consecutive scans of protein samples obtained on this high-flux beamline. Experiments have been designed to distinguish between two different possible mechanisms: (i) photoionization causing free radicals or secondary electrons producing degradation of the protein, and (ii) local heating of the sample resulting in protein denaturation. The latter appears to be the principal source of the signal deterioration.
\end{abstract}

Keywords: vacuum ultraviolet; circular dichroism spectroscopy; synchrotron radiation circular dichroism spectroscopy; protein structure; secondary structure; structural genomics; calibration; membrane protein; protein denaturation.

\section{Introduction}

Synchrotron radiation circular dichroism (SRCD) spectroscopy, first described more than 20 years ago (Sutherland et al., 1980; Snyder \& Rowe, 1980), has been used successfully in the ultraviolet (UV) and vacuum ultraviolet (VUV) wavelength ranges for structural analyses of macromolecules (Wallace, 2000). In first-generation SRCD stations, such as station 3.1 at the SRS and UV1 at ISA, the light intensity in the near-UV region is similar to that in a conventional circular dichroism (cCD) instrument, whilst the intensities of these beamlines in the wavelength range below $190 \mathrm{~nm}$ are several orders of magnitude higher than for a cCD instrument (Clarke et al., 2000; Wallace, 2000). In contrast, second-generation SRCD stations, such as CD12 at the SRS, have intensities of $\sim 10^{3}$ times those in CCD instruments even in the near-UV region (Clarke \& Jones, 2004). Because of these high intensities, SRCD provides a technique for which the signal-to-noise ratios, especially in the VUV, are greatly increased. Consequently, SRCD data can be collected to much lower wavelengths (below $160 \mathrm{~nm}$ ) (Wallace et al., 2004) providing a higher information content (Wallace \& Janes, 2001).
The ability to make reproducible measurements of $\mathrm{CD}$ spectra is of great importance for consistency as well as for obtaining proper secondary structure analyses. Spectra must be averaged in order to show reproducibility of spectral features and to estimate error levels, in order to discriminate between real and apparent differences. However, this procedure is reliant upon an absence of systematic changes between consecutive scans on the same sample. In a previous study, performed on the much lower intensity first-generation SRCD stations, it was found that even after irradiation for several hours no changes were seen in the SRCD spectra, nor were any changes found in the protein primary structure (Orry et al., 2001). However, using the more intense light available in a second-generation station we have found in the course of collecting a new reference data set of $\sim 70$ proteins (Lees et al., 2005) that in general the signal appears to degrade with each consecutive scan. There has been a suggestion that only single scans need be obtained for a given protein sample (Clarke \& Jones, 2004); however, such a procedure does not permit the examination of error levels or assessment of reproducibility.

Several mechanisms have been proposed to account for changes in the spectra upon irradiation in the VUV wave- 
length range: free-radical formation, which could lead to protein degradation (i.e. changes in the protein primary structure which in turn lead to secondary structural changes) or denaturation (local heating, which results in changes to secondary structure but not primary structure). In order to investigate the underlying mechanism for the observed signal deterioration (this paper) and to identify a means of circumventing it so that accurate reproducibility can be achieved [see also the accompanying paper by Janes \& Cuff (2005)], data were collected from several proteins, which differ in their chemical composition as well as in their sensitivity to VUV exposure. The results were compared with results from thermal denaturation studies, and the reversibility of the effects, as well as the chemical integrity of the proteins after irradiation, were examined.

\section{Materials and methods}

\subsection{Materials}

Horse myoglobin and jacalin were purchased from Calbiochem (USA); human serum albumin (HSA) was from Sigma Aldrich (UK). 3-Dehydroquinate dehydratase from Salmonella typhimurium (DHQase) was the gift of Professor Jeremy Lakey, University of Newcastle, and the translocator domain of the autotransporter NalP from Neisseria meningitidis was a gift from Dr C. J. Oomen, University of London and Utrecht University. All proteins were of $\geq 95 \%$ purity. Prior to use, unless otherwise noted all samples were extensively dialysed versus water using slide-A-lyzer MINI dialysis units (MW $10 \mathrm{~K}$ cut-off) from Pierce (UK), resulting in unbuffered samples. In a few cases, protein was examined in $100 \mathrm{~m} M$ phosphate buffer, $\mathrm{pH} 7.5$, or in $100 \mathrm{~m} M \mathrm{NaF}$, to determine the effects, if any, of buffering or high salt on protection from degradation effects. All samples were de-gassed under vacuum prior to use, and then centrifuged briefly to remove any undissolved material. Quantitative amino acid analyses were performed in duplicate at the Protein and Nucleic Acid Chemistry (PNAC) Facility located at the University of Cambridge, UK.

\subsection{Circular dichroism spectroscopy}

Except where otherwise noted, SRCD spectra were collected at station CD12, located at the SRS Daresbury Laboratory. After each beam refill (i.e. usually two times each day) the instrument was re-calibrated using camphor sulfonic acid (CSA) as described previously (Miles et al., 2003). No significant differences in CSA ratios were observed, which indicates the reproducibility of the beam characteristics in different fills.

Protein samples at $\sim 10 \mathrm{mg} \mathrm{ml}^{-1}$ protein (the final protein concentrations were determined according to quantitative amino acid analyses) were examined in a circular demountable $0.0015 \mathrm{~cm}$-pathlength Suprasil cell (Hellma UK), which had been previously calibrated using both interferometry and chromate dilution methods (Miles et al., 2005). Baseline spectra of the dialysates were measured under the same conditions as the samples. CD spectra were also collected on an Aviv 202 instrument, on SRCD station UV1 at ISA (Denmark) and on station U9b at the NSLS (USA) under similar conditions using the same cell, except in the case of the Aviv instrument where data were collected down to $185 \mathrm{~nm}$. Unless otherwise noted, spectra were measured at $277 \mathrm{~K}$.

For the thermal denaturation experiments, spectra were collected on station UV1 over the wavelength range 300$175 \mathrm{~nm}$ in $1 \mathrm{~nm}$ steps using $3 \mathrm{~s}$ dwell times with a bandwidth of $0.5 \mathrm{~nm}$. In successive scans, the temperature was incremented in $10^{\circ}$ intervals over the temperature range $278-358 \mathrm{~K}$, with equilibration times at each temperature of $5 \mathrm{~min}$ prior to scanning. A final spectrum was measured at $278 \mathrm{~K}$ after slowly lowering the temperature and allowing for refolding for at least $30 \mathrm{~min}$.

\subsection{Irradiation experiments}

2.3.1. Time-dependent changes. On station $\mathrm{CD} 12$, spectra were collected over the wavelength range $300-175 \mathrm{~nm}$ in $1 \mathrm{~nm}$ steps at $1 \mathrm{~s}$ dwell time (total integration) using a spectral bandwidth of $1 \mathrm{~nm}$ for 24 consecutive scans ( 5 min per scan) at $277 \mathrm{~K}$. In experiments to distinguish whether the exposure to the high-flux beam caused continued degradation even after exposure, consecutive scans were collected in a similar manner, except that the beam shutter was closed during every second scan. For the refolding experiments, after exposure the sample was stored at $277 \mathrm{~K}$ for $48 \mathrm{~h}$ and the spectrum recollected on a cCD instrument. To examine flux dependence, spectra were collected for HSA, myoglobin and jacalin at the lower-flux beamline at UV1, at U9b and/or on the cCD instrument using aliquots of the same (unirradiated) sample in the same sample cell and cell holder, and under similar conditions.

2.3.2. Wavelength-dependent changes. Separate identical freshly loaded samples of HSA, at $1 \mathrm{mg} \mathrm{ml}^{-1}$ concentration in a $0.01 \mathrm{~cm}$-pathlength cell, were exposed at 280, 240, 220, 175 and $160 \mathrm{~nm}$ for periods of between 15 and $75 \mathrm{~min}$ (in $15 \mathrm{~min}$ intervals). After exposure each sample was scanned once in the regions $195-185 \mathrm{~nm}$ and $217-227 \mathrm{~nm}$ to measure the differential effects on the peaks in these two regions separately. An action spectrum was calculated from these data using the initial rate constants at each wavelength of exposure.

\subsection{Measurements of sample integrity}

2.4.1. SDS polyacrylamide gel electrophoresis. $1 \mu \mathrm{l}$ samples of each protein both before and after exposure on CD12 (scanning 24 times between 280 and $175 \mathrm{~nm}$, for a total exposure of $3 \mathrm{~h}$ ) were run on a homogeneous $12.5 \%$ PHAST gel (Amersham Pharmacia Biotech) at $250 \mathrm{~V}, 10.0 \mathrm{~mA}, 298 \mathrm{~K}$ and stained with Coomassie blue. Molecular weight standards were from a low-molecular-weight calibration kit (Pharmacia).

2.4.2. Mass spectrometry. Mass spectra were collected on a Micromass platform single-quadrupole mass spectrometer (Micromass, UK). Samples of $10 \mu \mathrm{l}$ of proteins before and after exposure to the beam (as above) were injected under 
standard conditions (50\% acetonitrile $/ 0.25 \%$ formic acid) and the delivery solvent $(50 \%$ acetonitrile $)$ was pumped at $10 \mu \mathrm{min}^{-1}$. Twelve $10 \mathrm{~s}$ scans in positive-ion mode were accumulated for each sample over the $m / z$ range 750-1150. Spectra were processed using the Masslynx software supplied by Micromass (version 3.4).

\subsection{Secondary structural analyses}

Spectra were processed using CDtool software (Lees et al., 2004). Identification of principal eigenvectors contributing to each spectrum ('principal component analyses' or PCA) also used routines in the CDtool package. Secondary structure analyses were carried out using the DICHROWEB webserver (Whitmore \& Wallace, 2004) with the CONTINLL algorithm (Provencher \& Glockner, 1981; Van Stokkum et al., 1990) and reference data set 1 (Sreerama \& Woody, 2000). The goodness-of-fit parameter reported, NRMSD (Mao et al., 1982), is a measure of the correspondence of the experimental and calculated spectra, with small values corresponding to good correlations.

\section{Results}

\subsection{Choice of proteins}

We had observed spectral changes in successive scans for the vast majority of the $\sim 70$ proteins whose SRCD spectra we collected in the process of creating a new reference database for protein folds (Wallace et al., 2004; Wien et al., 2005). Several representative proteins were chosen for detailed study based on their different chemical and structural properties. The five proteins in this study (myoglobin, PDB code 1ymb; HSA, PDB code 1n $5 \mathrm{u}$; DHQase, PDB code 1qfe; jacalin, PDB code 1ku8; NalP, PDB code 1uyo) encompassed the following different characteristics: having no disulfide groups (myoglobin) and having a large number (17) of disulfide groups (HSA); mainly helical (HSA, myoglobin), mixed helical and sheet (DHQase) and mainly sheet (jacalin) proteins; single subunit (myoglobin) and multiple subunit (HSA, DHQase) proteins; proteins having high (17\% jacalin) and low (5.6\% DHQase) aromatic amino acid contents; soluble (myoglobin, jacalin, HSA, DHQase) and outer membrane (NalP) proteins. All spectra shown are for proteins in distilled water (in order to obtain the lowest-wavelength data possible), but studies have also been carried out in buffer (100 $\mathrm{m} M$ phosphate, $\mathrm{pH} 7.5)$ and high salt $(100 \mathrm{~m} M \mathrm{NaF}$ ) and exhibit similar behaviour (i.e. no enhanced stability) under these conditions. The five proteins exhibit behaviours typical of the types of behaviours we have seen for many other proteins when exposed to the high-flux VUV light. Many of the studies depicted in this paper were performed on HSA, a protein whose three-dimensional structure is known (Wardell et al., 2002) which shares $75 \%$ sequence identity with bovine serum albumin, a protein previously shown to be particularly susceptible to irradiation (Moriyama et al., 1994). Like its bovine homologue, HSA was found to be very susceptible to irradiation effects.

\subsection{Observed spectral changes}

Proteins which were affected by the high-flux VUV light demonstrated systematic changes to the spectral shape and magnitude during consecutive scans (Fig. 1a). No changes were seen in the accompanying baseline spectra (data not shown), so the differences were not simply a result of beam decay or other systematic instrumental changes with time. The typical radiation dose for a $5 \mathrm{~min}$ scan on CD12 would correspond to roughly $3 \mathrm{~kJ} \mathrm{~m}^{-2}$ (Clarke \& Jones, 2004). In equivalent experiments measuring consecutive scans on either lower-flux SRCD (Fig. 1b) or on the CCD, no changes were observed, suggesting the phenomenon is flux-dependent.

\subsection{Possible mechanisms}

Several possible mechanisms have been proposed for the types of deteriorations in signal observed (Orry et al., 2001).

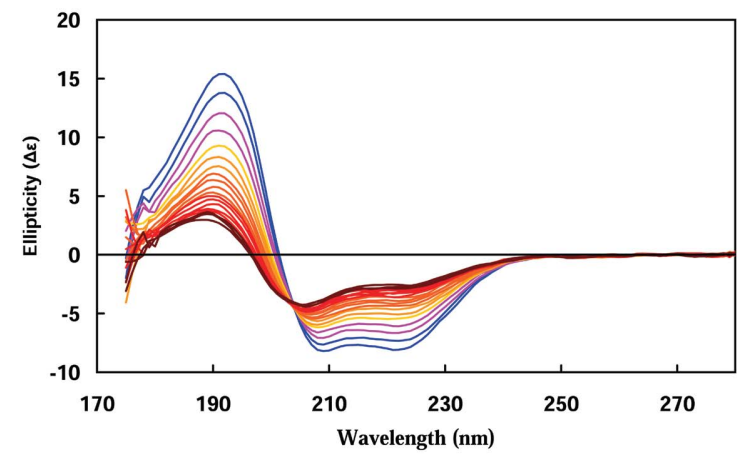

(a)

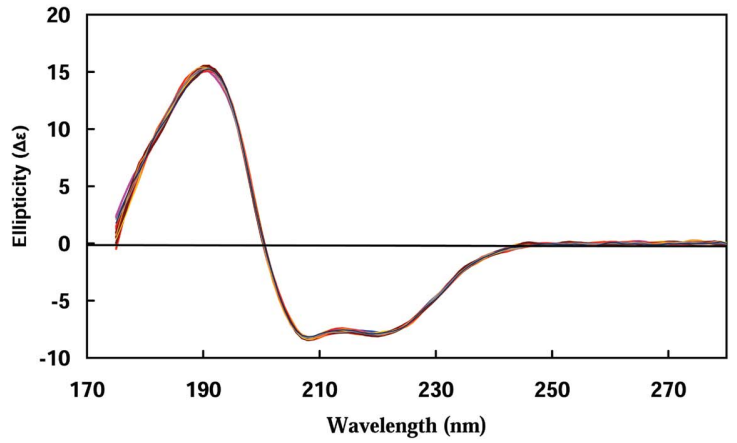

(b)

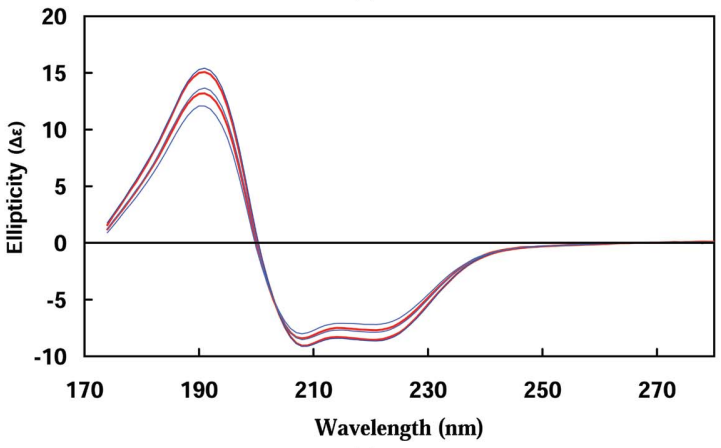

(c)

Figure 1

24 consecutive scans of HSA obtained $(a)$ at station CD12 at the SRS and (b) at station UV1 at ISA. (c) A comparison of three scans obtained on CD12 with the shutter open during each scan (blue), and scans 1 and 3 obtained with the shutter closed during scan 2 (red). 
The principal potential mechanisms are free-radical formation (Clarke \& Jones, 2004), which will contribute to protein degradation (and irreversible changes to both primary and secondary structure), and local heating in the sample causing protein denaturation (producing either reversible or irreversible changes to the secondary structure, but no changes in the primary structure). The denaturation could either be similar to that produced by the macroscopic heating of proteins in thermal denaturation experiments, or could be somewhat different, arising from specific heating of bound waters that preferentially denature different local regions of the protein. The goal of this study was to discriminate between all these possibilities, so studies were undertaken to determine the chemical integrity of the samples, the wavelength-dependence of the phenomenon, the nature of the changes in terms of secondary structure, the reversibility of the changes, and any correlation with thermal denaturation studies.

\subsection{Chemical integrity}

To ascertain if irradiation produced chemical changes, such as cleavage, cross-linking or chemical modifications in the proteins, we examined samples which had been extensively irradiated (to the point where their signals had degraded to $30 \%$ of the initial signal) and compared them with unirradiated samples. Using SDS gel electrophoresis to check for crosslinking and/or cleavage (Fig. 2) and mass spectrometry to check for modification or cleavage (Fig. 3), we found no detectable differences between the samples that had been exposed at a range of wavelengths and those that had not been exposed to the beam. Hence, these results are consistent with denaturation but not degradation, and are evidence that the free-radical mechanism does not dominate the processes which produce the spectral changes.

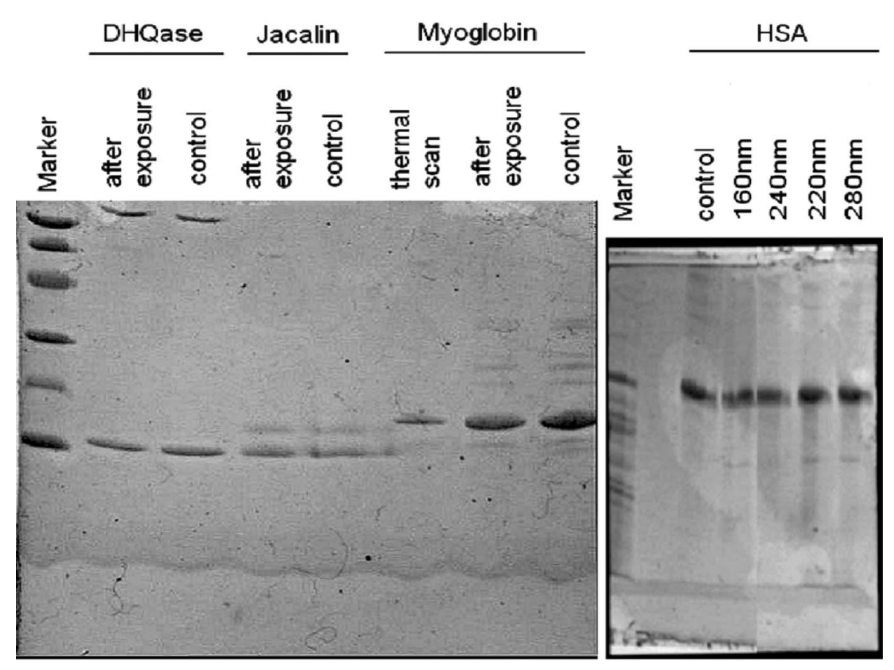

Figure 2

Left-hand panel: samples of DHQase, jacalin and myoglobin before exposure ('control') and after $2 \mathrm{~h}$ of exposure at station CD12. Righthand panel: HSA was inspected before ('control') and after irradiation for $2 \mathrm{~h}$ at four different wavelengths $(160,220,240$ and $280 \mathrm{~nm})$.
Table 1

Calculated secondary structure (\%) for the first and 25th scans of HSA.

\begin{tabular}{llrlll}
\hline Scan & Helix & Sheet & Turn & Other & NRMSD $\dagger$ \\
\hline 1 & 75 & 0 & 11 & 14 & 0.03 \\
25 & 26 & 17 & 24 & 32 & 0.1 \\
X-ray $\neq$ & 72 & 0 & 15 & 12 & \\
\hline
\end{tabular}

$\dagger$ NRMSD is a goodness-of-fit parameter. $\ddagger$ X-ray is the secondary structure found in the crystal structure.

\subsection{Secondary structural effects}

To examine what structural effects the spectral changes correspond to, we calculated the secondary structures of HSA based on the first and 25th scan (Table 1). The helical secondary structure content calculated for the first scan $(75 \%)$ correlates well with the secondary structure found in the crystal structure (72\%) (Wardell et al., 2002) (Table 1). However, the helix content calculated for the final scan is much lower than expected for this protein. Thus the changes which occurred correspond to a loss in helical content with a concomitant increase in other (or disordered) content, a result that is consistent with a denaturation mechanism. Furthermore, using principal component analyses and singular-value decomposition with the two major components, the degradation kinetics can be followed (Fig. 4). Whilst the initial spectrum is dominated by a component (Fig. 4a) that appears

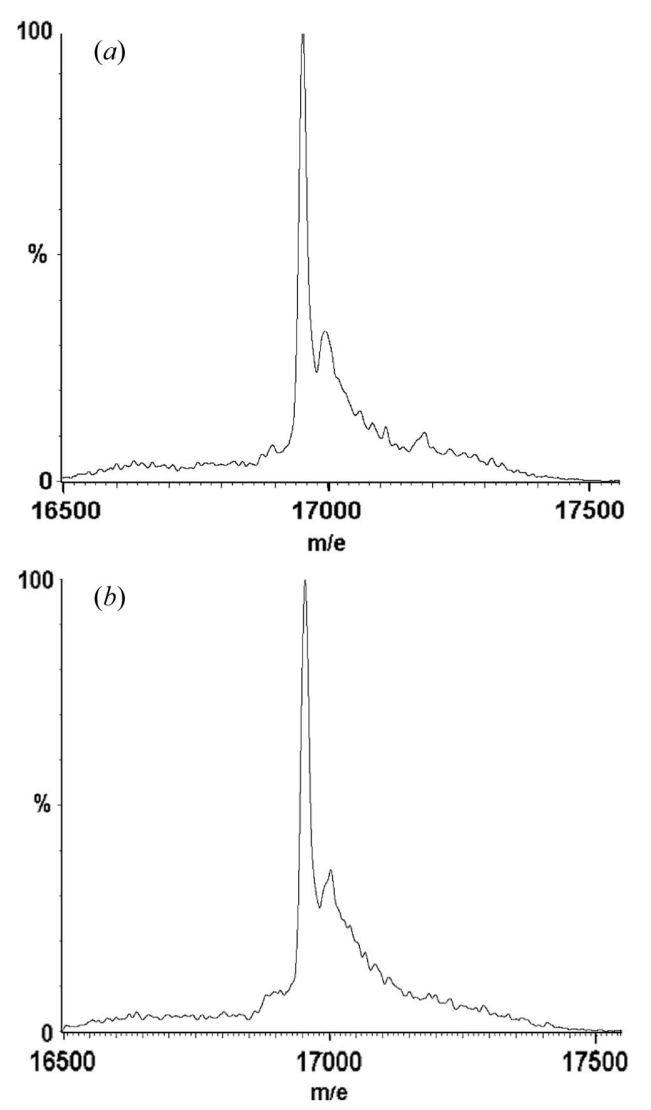

Figure 3

Mass spectra of myoglobin $(a)$ before and $(b)$ after $2 \mathrm{~h}$ continuous exposure on CD12. 


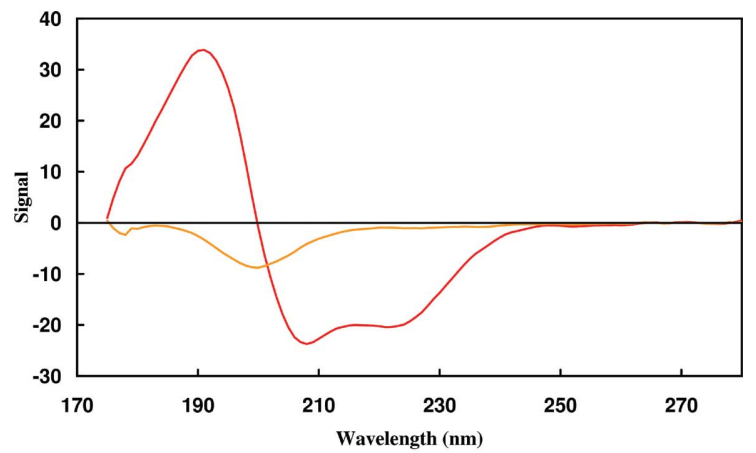

(a)

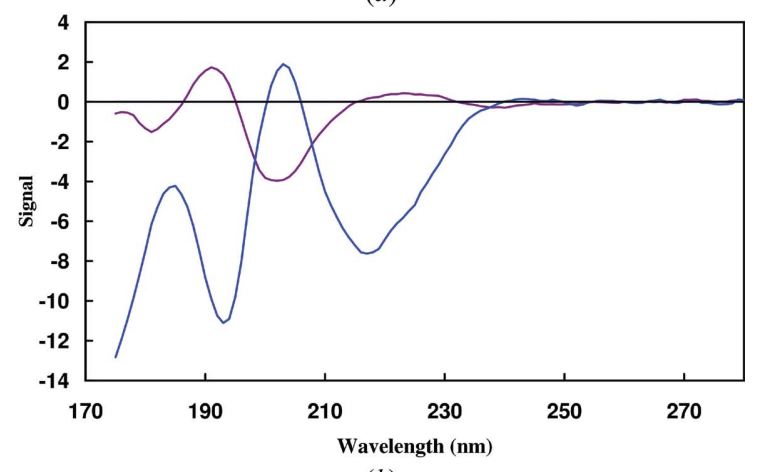

(b)

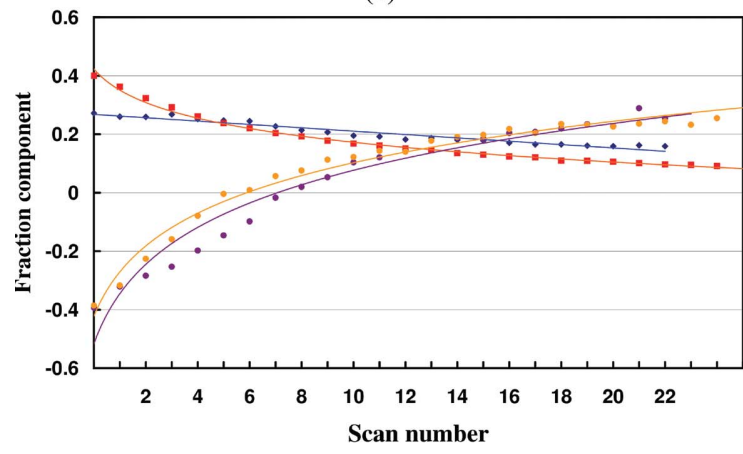

(c)

Figure 4

Principal component analyses showing the first and second basis spectra for $(a)$ HSA in red and orange, respectively, and $(b)$ jacalin in blue and purple, respectively. (c) The fractional contributions of the two principal basis spectra for each sample in consecutive scans [using the same colour schemas as in parts $(a)$ and $(b)]$.

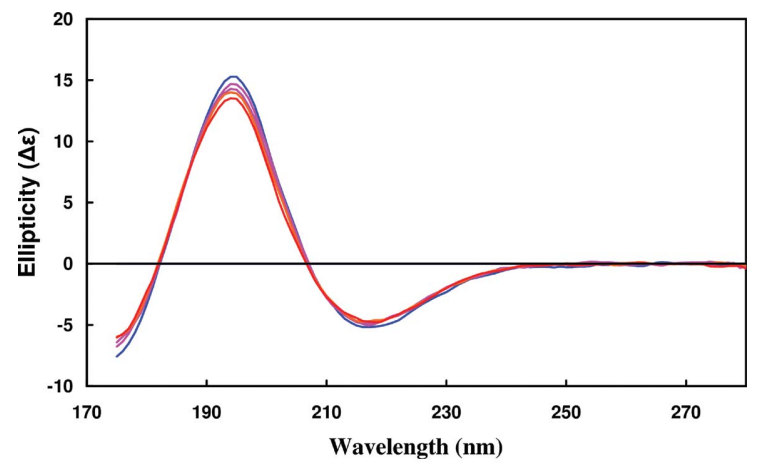

Figure 5

Five consecutive spectra obtained on CD12 for the membrane protein NalP, showing the lower sensitivity of this protein to irradiation. similar to a helical component (Fig. $4 a$, red curve), the changes plateau (Fig. 4c) to what appears to be a mostly disordered structure (Fig. 4a, orange curve), in a manner similar to our observations for the thermal denaturation process (see below). These results are not what would be expected from a degradation mechanism that cleaves peptide bonds. In that case, there should be a continuous loss in signal, perhaps accompanied by some conversion to disordered structure, but no plateau would be expected (i.e. degradation should continue until all molecules are destroyed). Hence, again, the observations in this study are more consistent with a denaturation mechanism.

\subsection{Differential effect with different proteins}

Degradation could potentially be related to the amino acid composition of a protein, especially if, as has been seen with higher-energy X-ray irradiation, it contains a large number of, for example, disulfide groups or aromatic amino acids $(\mathrm{Xu}$ et al., 2003) which are susceptible to free-radical attack. However, in the proteins specifically examined in this study, and in the larger set of proteins in our reference database, both proteins with and without disulfides, with high and low aromatic compositions, and monomeric and multimeric proteins were shown to undergo the effect. This could argue against specific types of degradation, but does not exclude a degradation mechanism mostly associated with cleavage of peptide bonds. The only class of proteins that consistently seems to be less affected is that of membrane proteins [both outer membrane proteins as in the example in Fig. 5, as well as inner (helical) membrane proteins (data not shown)].

\subsection{Wavelength dependence}

If the phenomenon observed is due to local heating of water molecules, it would be expected that there would be a strong wavelength dependence, peaking near the absorbance maximum of water. In this study we measured a (kinetic) action spectrum for the deterioration in two of the protein peaks (Fig. 6). Although we were only able to examine irradiation down to $175 \mathrm{~nm}$ due to absorption of the cells, the result is consistent with the action spectrum having a peak near the absorption peak of water at $\sim 166.5 \mathrm{~nm}$ (Chung et al., 2001); however, it could also be associated with the increasingly high-energy photons at low wavelengths causing more degradation. This issue is further addressed in the accompanying paper by Janes \& Cuff (2005).

\subsection{Time-dependent changes}

Experiments were carried out in which the shutter was closed during every other scan, thereby decreasing the irradiation by a half (Fig. 1c). In this case the deterioration appeared to occur at roughly half the rate, suggesting that the mechanism did not involve initial damage followed by migration of free radicals or secondary electrons to cause further damage. This result is consistent with a denaturation rather than degradation mechanism. 


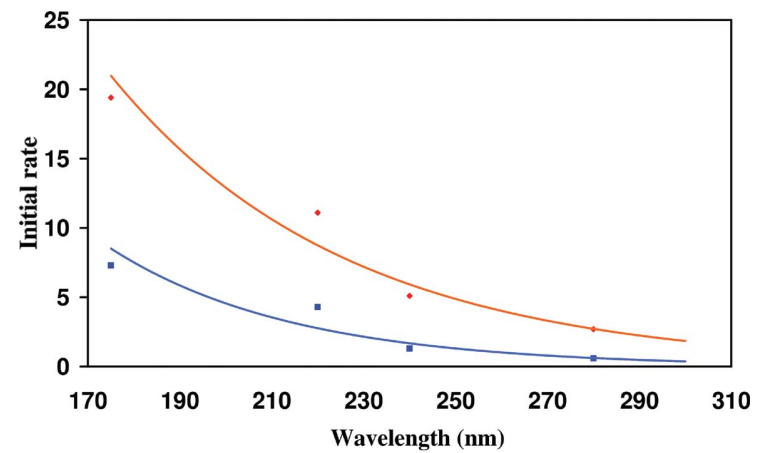

Figure 6

Action spectrum showing the rate of change of the signal as a function of wavelength of irradiation for the two peaks centred at $\sim 190 \mathrm{~nm}$ (red) and $\sim 224 \mathrm{~nm}$ (blue) for HSA on CD12.

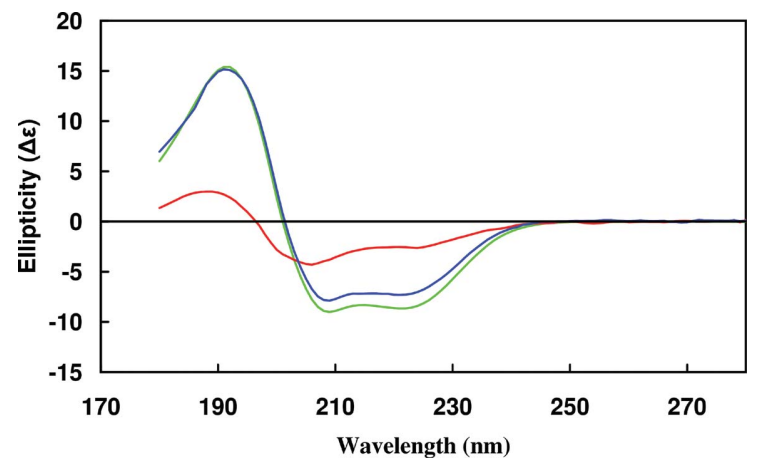

Figure 7

Spectra of the first HSA scan (green), of the 25th scan on CD12 (red) and of the sample after 'recovery' for $48 \mathrm{~h}$ (blue).

\subsection{Reversibility}

The reversibility experiments provide the most compelling evidence (other than the mass spectrometry) that chemical modification is not the principal mechanism for the deterioration. If molecules were 'damaged' by bond breaking they would not recover upon standing in the absence of the beam. If the mechanism was a form of denaturation, partial or total refolding could occur, depending on the extent and nature of the unfolding. Just as some proteins can refold after thermal denaturation, some proteins in this study could recover at least some of their structure after removing the beam and allowing the sample to refold with time (Fig. 7). In this study, not all proteins refolded, but then not all proteins can refold after thermal unfolding. However, the observation that at least some of them could partially refold is consistent only with a denaturation mechanism.

\subsection{Comparison with thermal denaturation}

To try to understand the denaturation mechanism, thermal denaturation studies were undertaken on a lower-flux beam (UV1 or the Aviv cCD instrument) where no flux-related deterioration is observed. The successive scans of HSA show changes in signal that are similar, but not identical, to those found in the high-flux irradiation scans (Fig. 8). They also exhibited generally similar PCA components and kinetics (data not shown). The seventh scan at high flux corresponds

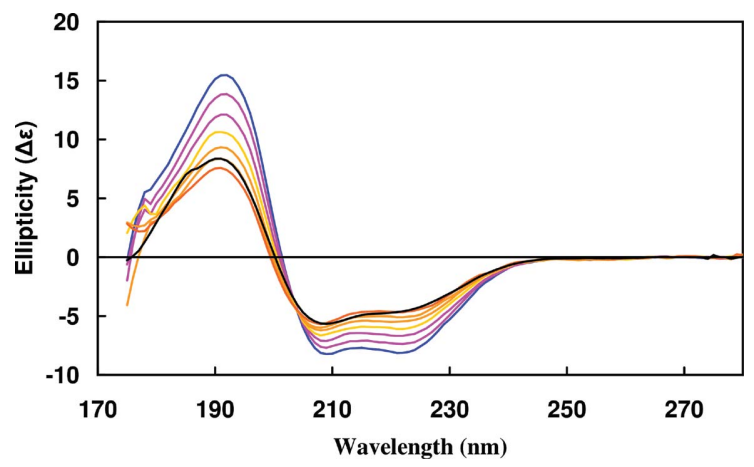

Figure 8

Seven consecutive scans on CD12 overlaid on the $358 \mathrm{~K}$ temperature scan (black) obtained at UV1.

roughly to the $358 \mathrm{~K}$ spectrum in the thermal denaturation scan. These results are consistent with a type of denaturation, but with a different order of denaturation of elements within the structure than seen in a bulk heating experiment. We propose that the differences in the kinetics of the two types of denaturation are due to the identity of the thermally excited molecules. In the former it may be the tightly bound waters that perturb specific elements of adjacent secondary structure, whilst in the latter it may be the bulk water that affects the protein stability.

\section{Discussion/conclusions}

In summary, a series of experiments were performed to distinguish between two possible mechanisms for the deterioration in signal observed in consecutive scans on a high-flux SRCD beamline. The data are consistent with the principal cause being denaturation owing to local heating, rather than degradation owing to free-radical formation, although we cannot completely rule the latter out as being a minor contributor to the process.

Whilst there was no detectable overall heating of the sample during the course of the experiment (as measured by a probe placed directly inside the sample cell), the heating could be specifically localized to microscopic regions within the sample. The accompanying paper by Janes \& Cuff (2005) also shows that the phenomenon is not due to the heating of the bulk water in the sample. We propose that it involves heating of water molecules which are either bound to or buried inside the protein, and thus form an integral part of the three-dimensional structure of the protein.

The changes in secondary structure observed following irradiation are similar, but not identical, to those seen in the thermal denaturation experiments. Molecular dynamics studies (Bizzarri \& Cannistraro, 2002) have shown that the buried waters have different thermal susceptibilities than do bulk water, and hence they may be preferentially heated by the energy of the incoming light. This explanation is also consistent with the observation that membrane proteins seem to be less affected than are soluble proteins, since membrane proteins tend to have fewer buried waters than do soluble proteins. 


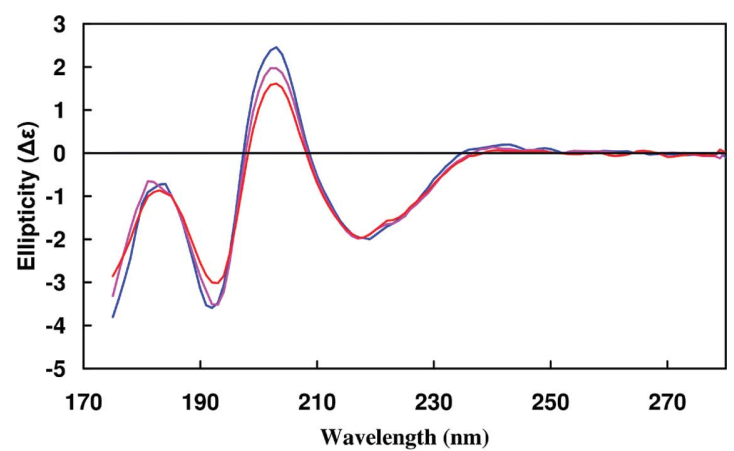

(a)

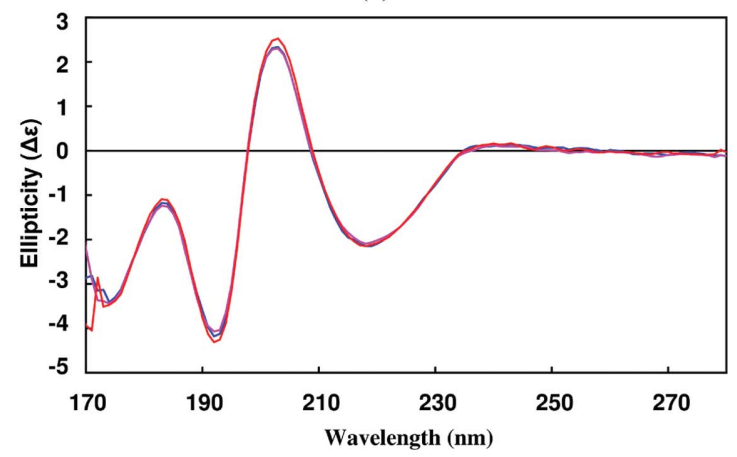

(b)

Figure 9

Three successive scans of jacalin $(a)$ taken consecutively on the same sample and $(b)$ on separately loaded samples.

Having identified a probable source of the signal deterioration, it then becomes important to devise methods that will eliminate such artifacts in repeated scans. There appear to be at least two suitable means of overcoming the problem: (i) replace the sample in successive scans with a fresh sample (Fig. 9) [this method requires a high reproducibility in loading and hence in the design of the cells and sample holders (Wien \& Wallace, 2005) and is obviously demanding on both time and sample], and (ii) as described in the accompanying paper by Janes \& Cuff (2005), the effect can also be eliminated (perhaps more facilely) by decreasing the beam size, thereby reducing the flux and only permitting irradiation of a small portion of the sample, which allows diffusion of the small fraction of proteins that are 'damaged' out of the beam. Hence, using either of these methods, it should be possible, even using high-flux beamlines, to produce reproducible spectra in which systematic degradation effects do not adversely affect the results obtained.

This work was supported by grants B13586 and B14225 from the BBSRC to BAW and grants from the CLRC to BAW for beam time at the SRS. Beam-time access at ISA was enabled by the European Community - Research Infrastructure Action under the FP6 'Structuring the European Research Area' Programme to Søren Pape Møller (Aarhus University). Some research was carried out at the National Synchrotron Light Source, Brookhaven National Laboratory, which is supported by the US Department of Energy, Division of Materials Sciences and Division of Chemical Sciences, under Contract No. DE-AC02-98CH10886. JGL was supported by a BBSRC CASE studentship with GlaxoSmithKline, and AJM was supported by an MRC studentship. We thank Dr Robert W. Janes (Queen Mary, London) for helpful discussions, and John Trunk (Brookhaven National Laboratory) for technical assistance on beamline U9b. We also thank Dr Robert Sarra (Birkbeck) for help with the mass spectrometry and Peter Sharratt (PNAC, Cambridge) for the QAA analyses.

\section{References}

Bizzarri, A. R. \& Cannistraro, S. (2002). J. Phys. Chem. B, 106, $6617-$ 6633.

Chung, C. Y., Chew, E. P., Cheng, B. M., Bahou, M. \& Lee, Y. P. (2001). Nucl. Instrum. Methods, 467, 1572-1576.

Clarke, D. T., Bowler, M. A., Fell, B. D., Flaherty, J. V., Grant, A. F., Jones, G. R., Martin-Fernandez, M. L., Shaw, D. A., Todd, B., Wallace, B. A. \& Towns-Andrews, E. (2000). Synchrotron Rad. News, 13, 21-27.

Clarke, D. T. \& Jones, G. R. (2004). J. Synchrotron Rad. 11, 142-149. Janes, R. W. \& Cuff, A. J. (2005). J. Synchrotron Rad. 12, 524-529.

Lees, J. G., Smith, B. R., Wien, F., Miles, A. J. \& Wallace, B. A. (2004). Anal. Biochem. 332, 285-289.

Lees, J. G., Wien, F., Miles, A. J. \& Wallace, B. A. (2005). In preparation.

Mao, D., Wachter, E. \& Wallace, B. A. (1982). Biochemistry, 21, 49604968.

Miles, A. J., Wien, F., Lees, J. G., Rodger, A., Janes, R. W. \& Wallace, B. A. (2003). Spectroscopy, 17, 653-661.

Miles, A. J., Wien, F., Lees, J. G. \& Wallace, B. A. (2005). Spectroscopy, 19, 43-51.

Moriyama, Y., Kakehi, T. \& Takeda, K. (1994). Anal. Biochem. 219, 378-380.

Orry, A. J. W., Janes, R. W., Sarra, R., Hanlon, M. R. \& Wallace, B. A. (2001). J. Synchrotron Rad. 8, 1027-1029.

Provencher, S. W. \& Glockner, J. (1981). Biochemistry, 20, 33-37.

Snyder, P. A. \& Rowe, E. M. (1980). Nucl. Instrum. Methods, 172, 345-349.

Sreerama, N. \& Woody, R. W. (2000). Anal. Biochem. 287, 252-260.

Sutherland, J. C., Desmond, E. J. \& Takacs, P. Z. (1980). Nucl. Instrum. Methods, 172, 195-199.

Van Stokkum, I. H. M., Spoelder, H. J. W., Bloemendal, M., Van Grondelle, R. \& Groen, F. C. A. (1990). Anal. Biochem. 191, 110 118.

Wallace, B. A. (2000). J. Synchrotron Rad. 7, 289-295.

Wallace, B. A. \& Janes, R. W. (2001). Curr. Opin. Chem. Biol. 5, $567-$ 571.

Wallace, B. A., Wien, F., Miles, A. J., Lees, J. G., Hoffman, S. V., Evans, P., Wistow, G. J. \& Slingsby, C. (2004). Faraday Discuss. 126, 237243.

Wardell, M., Wang, Z., Ho, J. X., Robert, J., Ruker, F., Ruble, J. \& Carter, D. C. (2002). Biochem. Biophys. Res. Commun. 291, 813819.

Whitmore, L. \& Wallace, B. A. (2004). Nucl. Acids Res. 32, W668W673.

Wien, F., Miles, A. J., Lees, J., Cuff, A. L., Janes, R. W. \& Wallace, B. A. (2005). Biophys. J. 88, 2727a.

Wien, F. \& Wallace, B. A. (2005). Submitted.

Xu, G. Z., Takamoto, K., Guan, J. Q. \& Chance, M. R. (2003). Biophys. J. 84, $36 a$. 